\title{
Hematometra following caesarean section for placenta previa
}

\author{
S Gupta, MBBS, DNB (O\&G); M Tyagi, MBBS, MD; E Khanuja, MBBS, MS D Prakash, MBBS \\ Department of Obstetrics and Gynaecology, Subharti Medical College, Meerut, Uttar Pradesh, India
}

Corresponding author: S Gupta (gupta_smriti_80@yahoo.in)

Hematometra following caesarean section is rare. We present here a case of 23-year-old woman who had undergone caesarean section for placenta previa. She presented with cryptomenorrhoea and hematometra after 5 months of caesarean delivery owing to adhesions in the vagina. The hematometra was drained vaginally.

S Afr J Obstet Gynaecol 2017;23(3):76-77. DOI:10.7196/SAJOG.2017.v23i3.1148

Hematometra may be congenital or acquired. Acquired causes include senile atrophy of the endocervical canal, scarring of the isthmus by synechiae, radiation and endocervical malignancy or due to surgical procedures such as cone biopsy. Hematometra following a cesarean delivery is rare. ${ }^{[1]}$ We present a rare case of haematometra following caesarean section.

In October 2016, a 23-year-old patient, P1L1, presented to Subharti Medical College, Meerut, India, with abdominal pain for the previous 3 months. She gave a history of having undergone a caesarean section in June 2016 for placenta previa. Her postoperative period was uneventful and she passed lochia normally. She had not had a fever or a foul-smelling discharge per vaginam in the postoperative period. She developed pain in the abdomen over the previous 3 months that was cyclical, recurring at approximately the same time every month, abrupt in onset, severe and progressive in nature and lasted for 3 to 4 days each time. It was associated with spotting per vaginam only in the first month.

On examination, she was afebrile and her vitals were stable. Systemic examination was within normal limits. On per abdominal examination the uterus was enlarged corresponding to 14 weeks' size and was slightly tender. On speculum examination, all the fornices were obliterated and the cervix could not be visualised. On vaginal examination, the cervix could not be palpated but the uterus was enlarged as described. On rectal examination, the per vaginal findings were confirmed and the lower part of the uterus was found to be dilated and ballooned.

The urine pregnancy test was negative. and her full blood count was within normal limits. A large collection of $377 \mathrm{~cm}^{3}$ was noted on ultrasonography in the endometrial cavity extending into endocervical canal, which was suggestive of hematometra. Under spinal anaesthesia, the vagina was visualised with the help of two Sims' posterior vaginal wall specula. As the cervix was not visualised, the cervix could not be held with any instrument but gentle pressure was applied with a Hegar dilator at the apex of the visible vagina. The motion was transmitted to the fundus of the uterus and could be felt abdominally. The Hegar dilator was pushed gently in this area in an upward and anterior direction. While doing so, the external os could be negotiated and $\sim 300 \mathrm{~cm}^{3}$ of old collected blood was drained from the endometrial cavity. The cervical canal was then dilated serially with Hegar dilators up to number 8 to prevent future restenosis.
There were no intrauterine adhesions. Post procedure, the vaginal and rectal examinations revealed that the size of the uterus had reduced to 8 weeks. The patient was administered intravenous antibiotics and discharged on the third postoperative day.

Within 2 weeks of draining the hematometra, the patient resumed her menstrual cycle with a normal flow of 3 days. She was relieved of cyclical abdominal pain. A follow-up scan showed no intrauterine collection. The patient was followed up for 3 months after the procedure and she continued to have menstrual cycles with normal flow.

\section{Discussion}

Cases of post-caesarean hematometra are rare. A more probable cause is either intrauterine adhesions or the internal os of the cervix may be found to be stenosed. Our patient had undergone caesarean delivery for placenta previa. Multiple hemostatic sutures during cesarean section for placenta previa have been demonstrated to be an important causative factor in hematometra formation. ${ }^{[3]}$ It can only be assumed that in our patient, deep sutures were taken in the placental bed in the lower uterine segment for haemostasis which accidently resulted in obliteration of the vaginal fornices. The patient had normal lochia in the postoperative period. But these deep sutures might have healed by fibrosis, as the cervix was obscured. This resulted in obliteration of the external os of cervix in the late post-operative period and hence formation of hematometra on resumption of menstruation.

In an attempt to achieve haemostasis during caesarean section, a number of innovative techniques have been tried apart from the usual method of oversewing the open bleeding sinuses on the lower segment. None of the methods have been known to cause postoperative adhesions, hematometra or amenorrhoea. When we are confronted with a patient with placenta previa experiencing massive haemorrhage during caesarean delivery, haemostasis is first attempted using uterotonic drugs, uterine massage and intrauterine packing. However, if these manoeuvres fail, a number of surgical techniques, such as uterine artery ligation, hypogastric artery ligation and uterine compression sutures have been described. ${ }^{[3]}$

Treatment of cervical stenosis is indicated only if symptoms or uterine abnormalities are present and may involve cervical dilatation. ${ }^{[2]}$ In our case, cervical dilatation was performed with 


\section{CASE REPORT}

Hegar dilators and the patient did not immediately require repeated dilatation. Further follow-up of the patient is required.

Acknowledgements. None.

Author contributions. SG and MT: diagnosis and surgical management. EK and DP: helped with patient management. All authors contributed to the writing of the manuscript.

Funding. None.

Conflicts of interest. None.
1. Kaur G, Jain S, Sharma A, Vaid NB. Hematometra formation-A rare comlication of cesarean delivery. J Clin Diag Res 2014;8(8):OD03-OD04. https://doi.org/10.7860/JCDR/2014/8364.4689

2. Thomas S, Roy P, Biswas B, Jose R. Complete cervical stenosis following cesarean section and VVF repair. J Obstet Gynaecol India 2012;62(Suppl 1):49-51. https://doi.org/10.1007/s13224-013-0380-8

3. Poothavelil MB, Hamidi I, Hamidi G. Occlusion of upper genital tract following lower segment cesarean section for placenta praevia. Sultan Qaboos Univ Med J 2008;8(2):215-218

Accepted 8 December 2017 